\title{
Search for nuclearites with the ANTARES neutrino telescope
}

\author{
Gabriela Emilia Păvălaş* on behalf of the ANTARES Collaboration \\ Institute of Space Science, 409 Atomistilor Street, Magurele, Ilfov, 077125, Romania
}

E-mail: gpavalasespacescience.ro

\begin{abstract}
About thirty years ago, strange quark matter (SQM) was hypothesized to be the ground state of hadronic matter and was also suggested as a cold dark matter candidate. Although there is no experimental or astrophysical evidence yet for its existence, SQM may be present in the cosmic radiation as relic particles of the early Universe, or as fragments released in binary strange star collisions or supernovae. The ANTARES neutrino telescope is sensitive to massive and stable SQM particles, called nuclearites. Their velocity is assumed to be $\beta \sim 10^{-3}$, typical of objects gravitationally trapped inside the galaxy. Nuclearites reaching the ANTARES depth would yield a large amount of light to the detector, by means of blackbody radiation emitted by the heated water molecules along their path. A dedicated analysis will be presented, as well as the ANTARES sensitivity for a flux of downgoing nuclearites, using data taken in 2009.
\end{abstract}

The 34th International Cosmic Ray Conference,

30 July- 6 August, 2015

The Hague, The Netherlands

\footnotetext{
* Speaker.
} 


\section{Introduction}

The nature of dark matter, representing about $85 \%$ of the mass of the observable Universe, is an open question of today's physics. One of the hypothesized constituents of the dark matter is strange quark matter (SQM) [1], that may be present in cosmic radiation. Nuclearites are massive and stable lumps of SQM, composed of nearly equal numbers of up, down and strange quarks. They would interact with the ambient atoms by means of elastic or quasi-elastic collisions, displacing the atoms of matter in their path. Among relevant searches for nuclearites, the MACRO (Monopole, Astrophyics and Cosmic Ray Laboratory) experiment has set an upper limit for a downgoing nuclearite flux of 5.4 $10^{-16} \mathrm{~cm}^{-2} \mathrm{~s}^{-1} \mathrm{sr}^{-1}$ (90\% C.L.) [2], for nuclearites of mass $M_{N}>10^{14} \mathrm{GeV}$, while the SLIM (Search for LIght Monopoles) high altitude experiment in Bolivia has set flux upper limits of $1.3 \cdot 10^{-15} \mathrm{~cm}^{-2} \mathrm{~s}^{-1} \mathrm{sr}^{-1}$ (90\% C.L.) [3], for nuclearite masses $M_{N}>10^{10} \mathrm{GeV}$.

The ANTARES neutrino experiment, currently operating in the Mediterranean Sea, is also sensitive to the signal of non-relativistic nuclearites. These particles would yield a large signal inside the detector, by means of the blackbody radiation emitted along their path. Partial results on the search for nuclearites with ANTARES were reported in [4]. In the following, this work describes the characteristics of nuclearites, presents the analysis performed on the selected ANTARES data and the preliminary results obtained.

\section{Nuclearites}

According to Witten [1], SQM lumps could be stable at zero temperature and pressure, knowing that in a three-flavor quark system, the Fermi energy and subsequently the mass of the quark bag are reduced by the third flavor added, compared to a two-flavor system (made of up and down quarks). Moreover, phenomenological models indicate that lumps of SQM are stable and metastable for a wide range of strong interaction parameters [5].

Nuclearites, heavy lumps of strange quark matter, would be electrically neutral; the small positive electric charge of the quark core would be neutralized by electrons, either in weak equilibrium inside the SQM, or forming an extended electronic cloud. Since direct nuclear interactions with the atoms they encounter are prevented by Coulomb repulsion, the relevant interaction mechanism of nuclearites is represented by elastic collisions [6]. The rate of energy loss is then given by:

$$
\frac{d E}{d x}=-\sigma \rho v^{2}
$$

where $\rho$ is the density of the medium, $v$ is the nuclearite velocity and $\sigma$ its geometrical cross section:

$$
\sigma= \begin{cases}\pi\left(3 M / 4 \pi \rho_{N}\right)^{2 / 3} & \text { for } M \geq 8.4 \cdot 10^{14} \mathrm{GeV} \\ \pi \cdot 10^{-16} \mathrm{~cm}^{2} & \text { for lower masses }\end{cases}
$$

with a SQM density $\rho_{N}=3.6 \cdot 10^{14} \mathrm{~g} \mathrm{~cm}^{-3}$.

The propagation of nuclearites in sea water is described by the equation:

$$
v(L)=v_{0} e^{-\frac{\sigma}{M} \int_{0}^{L} \rho d x},
$$


where $\rho=1 \mathrm{~g} \mathrm{~cm}^{-3}$, and $v_{0}$ is the nuclearite speed at the Earth surface. The nuclearite collides with the atoms of water, giving them velocities of order $O\left(v_{0}\right)$. The temperature of the medium rises to $T \sim O(k e V)$ and a hot plasma is formed that moves outwards as a shock wave. The luminous efficiency (defined as the fraction of dissipated energy appearing as light) was estimated, in the case of water, to be $\eta \simeq 3 \cdot 10^{-5}[6]$.

\section{Analysis}

For the present analysis, a selection of the ANTARES data from 2009 was made, considering runs that satisfy a set of quality criteria, and a well calibrated detector. The analysis complies with the blinding policy of ANTARES, that requires the optimization of the method by using simulated events and data-MC comparisons for a fraction of the selected data. For this search, a fraction equivalent to $\sim 13$ days of data acquisition was used, containing runs ending in " 0 ".

In what concerns the signal, nuclearite events were simulated with a dedicated Monte Carlo code, that is briefly described in the section below, with the following masses: $10^{14}, 10^{15}, 10^{16}$, and $10^{17} \mathrm{GeV}$. As for the physics background, downgoing atmospheric muons files simulated with the MUPAGE code [7] were used.

Besides muons, bioluminescence is also present in the deep sea environment of the ANTARES detector. Bioluminescence background causes sporadic peaks in the singles rate of up to several $\mathrm{MHz}$ during periods of a few seconds or less, that mimic at a certain extent the nuclearite signal. Short bursts can appear during data taking in relatively good conditions, on time scales of the order of a frame, i.e. $\sim 104 \mathrm{~ms}$. Programs that provide the count rate on a reduced time scale, the trigger type during particular frames and the event display are used to identify these bursts. Bioluminescence is usually localized in a region of the detector, and persists for the duration of one or more consecutive frames.

The simulated nuclearite and atmospheric muon files were then processed with a program that uses the standard muon triggers and the charge thresholds corresponding to the considered period of operation. The optical background was added from each data run in the sample to the simulated hits from nuclearite and muon events, providing a so-called run by run simulation.

The standard muon triggers of ANTARES used in this analysis are the so-called directional trigger and cluster trigger. The directional trigger requires five local coincidences causally connected, within a time window of $2.2 \mu \mathrm{s}$. The cluster trigger requires two coincidences between two L1 hits ${ }^{1}$ in adjacent or next-to-adjacent storeys. When a muon event is triggered, all PMT pulses are recorded over $4 \mu \mathrm{s}$ in a snapshot. When two or more events have some overlapping hits, a merger of the events proceeds and a larger snapshot results.

In the following, the nuclearite simulation is briefly described, as well as the effects of the trigger processing on the simulated nuclearite events. Then, the reconstruction procedure and the selection conditions applied to the data and MC events are presented, followed by the preliminary results on the detector sensitivity.

\footnotetext{
${ }^{1}$ A local coincidence L1 is obtained when at least two L0 hits (hits with charge threshold $>0.3$ photo-electrons) occur within $20 \mathrm{~ns}$ on two different PMTs in the same storey or when a large charge hit occurs. The threshold for large hits usually corresponds to 3 photo-electrons.
} 


\subsection{Nuclearite simulation}

Nuclearites are simulated with a Monte Carlo program, that includes the propagation of the nuclearites through the Earth's atmosphere and sea water, as well as the simulation of the expected signal at the detector level. The main assumptions of the nuclearite simulation are the following: isotropic flux above the Earth's atmosphere, galactic velocities of $\beta=10^{-3}$ at the entrance in the atmosphere (50 km above sea level), the trajectory is a straight line, since the influence of gravity is negligible, the propagation in the atmosphere and sea water, the light yield at the detector level are calculated based on the phenomenological model proposed in [6].

Given that only nuclearites with masses larger than about $10^{22} \mathrm{GeV}$ are able to cross the Earth, and that the nuclearite flux in cosmic rays is expected to be decreasing with increasing nuclearite mass (as for heavy nuclei), only downgoing nuclearites were considered in this analysis.

A hemispherical volume of $548 \mathrm{~m}$ radius symmetrically surrounding the 12 line detector is used to generate and trace the nuclearites trajectories. The base of the hemisphere is placed on the sea bed, $100 \mathrm{~m}$ below the plane of the lowest ANTARES storeys, and with the pole on the ANTARES symmetry axis, $100 \mathrm{~m}$ above the plane of the highest storeys. The entry point of the nuclearite trajectory is generated on the surface of the hemisphere, having the coordinates $\left(x_{0}, y_{0}, z_{0}\right)$. The direction of the trajectory is then given by randomly generated zenith and azimuth angles. In order to simulate downgoing trajectories, a subroutine checks if the trajectory intersects the hemisphere at a point higher than the initial entry point; if true, the upper point becomes the entry point. Above the fiducial hemisphere, the path length of the nuclearite is computed by considering that the detector lies on a solid sphere with a radius equal to the Earth's radius, covered uniformly by a layer of water and by an outer layer representing the atmosphere. Both the propagation of nuclearites in the atmosphere and in the sea water are described by Equation 2.2.

In order to propagate the nuclearites through the hemisphere, the algorithm implements the energy loss mechanism presented in Section 2, and evaluates the position, the velocity $\beta$ and the number of hits on the $\mathrm{OM}$ in time steps of $2 \mathrm{~ns}$. The procedure is repeated until the nuclearite optical energy loss (integrated over the time step) is lower than $3 \mathrm{eV}$, or the nuclearite reaches the sea floor.

Regarding the effect of the muon triggers on nuclearites, unlike the muon events that are encapsulated in one snapshot, most nuclearite events result in a series of connected snapshots of variable durations. The duration of a snapshot depends on the light yield of the particle, and on

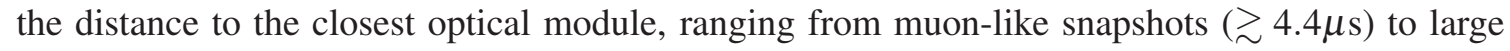
snapshots of up to few ms, produced by merging.

\subsection{Reconstruction and first level cuts}

The reconstruction of nuclearite trajectories uses the charge barycenter distribution as a function of time of the hits. Since the light emitted by nuclearites is isotropic, the charge barycenter gives an estimate of the position of the source at a certain moment. In case of a nuclearite passing through the detector, the displacement of the charge barycenter would indicate a downgoing track with a speed less than $10^{-3} \mathrm{c}$. The procedure consists in retrieving the time, charge and position of OMs for the hits of each event, and distribute them in time histograms of $500 \mathrm{~ns}$ bins. All hits with the charge $q>0.3$ p.e. are considered in the distributions. The time histograms of the charge 
barycenter projected on every axis are obtained from the following ratio, computed on each $500 \mathrm{~ns}$ bin:

$$
\frac{\sum q_{i} \cdot \operatorname{pos}_{i}}{\sum q_{i}}
$$

where $\operatorname{pos}_{i}=x, y, z$ is the position of the OM where the signal is detected and $i=1,2 . ., n$ is the number of hits in each bin.

The trajectory of the nuclearite is assumed to be linear, therefore the evolution in time of the charge barycenter distributions will be approximated by a straight line. The partial mean velocities $v_{x}, v_{y}, v_{z}$ at the detector level, as well as their errors, are determined from linear fits of the charge barycenter distribution on each axis as a function of time. Then, the total velocity and the corresponding uncertainty are obtained in a straightforward manner. The zenith angle and its uncertainty are determined as follows:

$$
\begin{gathered}
\theta=\arccos \left(v_{z} / v\right) \\
d \theta=\frac{1}{\sqrt{1-\left(\frac{v_{z}}{v}\right)^{2}}} \cdot \sqrt{\left(\frac{d v \cdot v_{z}}{v^{2}}\right)^{2}+\left(\frac{d v_{z}}{v}\right)^{2}} .
\end{gathered}
$$
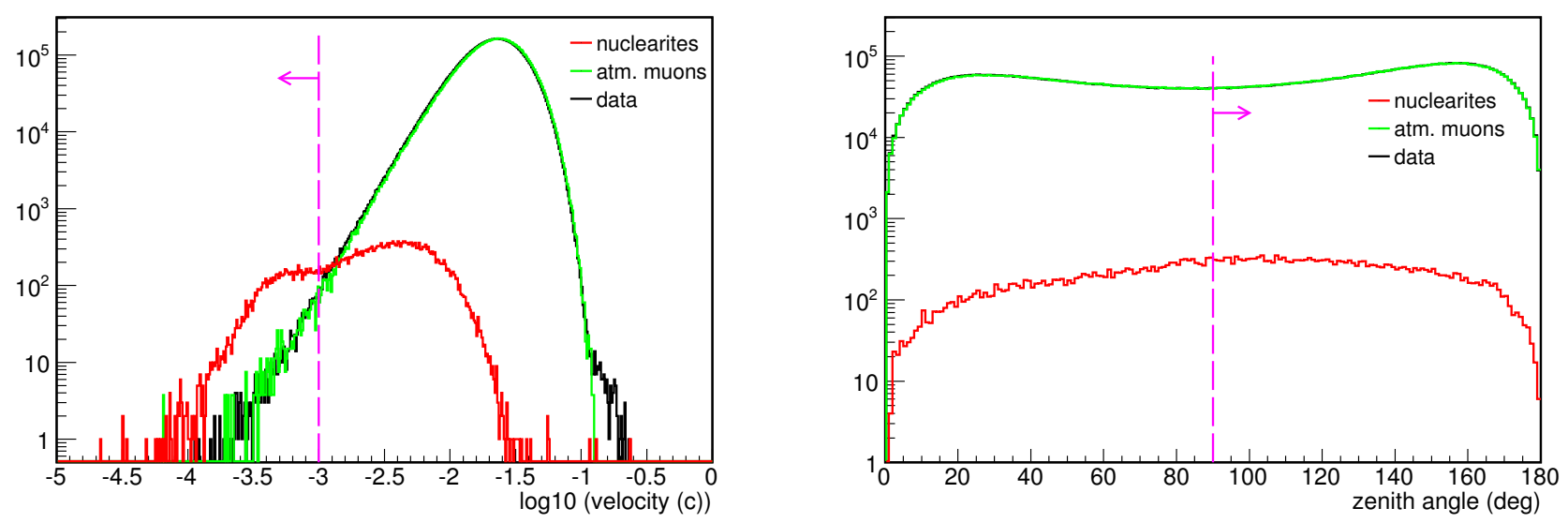

Figure 1: Left: Reconstructed velocity distributions for MC nuclearite snapshots, MC muons and data sample, with first level cut corresponding to $v<10^{-3} c$. Right: Reconstructed zenith angle distributions with cut represented by the vertical line.

The reconstruction procedure described above was applied to the selected data sample, to MC muons and nuclearite snapshots. The distributions of logarithmic reconstructed velocity and of zenith angle for data, MC muons and nuclearite snapshots are shown in Figure 1, with MC muon sample normalized to data. In what concerns data-MC comparison, a reasonable agreement is observed in the velocity distribution, except for the right tail of the distribution, where an excess of events is seen in data. In the low velocity region $\left(v<10^{-2} c\right)$, contribution from bioluminescence is also expected. The data-MC agreement of the zenith angle distribution is good. First level selection conditions are then defined, requiring a reconstructed velocity $v<10^{-3} c$ and a zenith angle $\theta>90^{\circ}$, consistent with the expected characteristics of nuclearite events, i.e. non-relativistic velocities and downgoing directions. 


\subsection{Second level cuts}

For the next step of the analysis, a number of discriminants was studied in order to select, from the snapshots surviving the first level cuts, the ones that might be part of a nuclearite event. These discriminants were the duration of snapshot, the number of L0 hits, and the number of L1 hits. The best discriminant for this analysis proved to be the number of L0 hits. The distributions of the logarithmic number of L0 hits for nuclearite, muon and data snapshots surviving the first level cuts are shown in Figure 2, on the left-hand side, with MC muons normalized to data distribution. At this stage, muon and data distributions do not agree well, since several snapshots with large values are observed only in data. These snapshots were found to belong to several frames in two runs, 39360 and 39680, and are shown in Figure 2 with blue line. They were investigated with tools dedicated to the bioluminescence identification, described at the begining of Section 3. The investigation indicates a bioluminescence origin of these snapshots. The number of all snapshots (including the ones triggered by one-dimensional selection algorithms) found in these frames is greater than the one usually seen in the quality runs, as shown in the right-hand side of Figure 2. In order to reject the suspicious snapshots, a selection cut at 200 snapshots, denoted C2a, was applied to the number of snapshots found in frames of data, as well as to the number of snapshots produced by nuclearite events.

After the removal of the noise, the muon and data distributions are in a reasonable agreement. In order to obtain the best sensitivity for the detector, the cut on the number of L0 hits was optimized. The best sensitivity is obtained by minimizing the so-called Model Rejection Factor [8], $\mathrm{MRF}=\frac{\bar{\mu}_{90 \%}\left(n_{b}\right)}{n_{s}}$, where $\bar{\mu}_{90 \%}$ is the "average upper limit" that would be observed by an ensemble of hypothetical experiments with no true signal and expected background $n_{b}$. The $\bar{\mu}_{90 \%}$ factor is taken from the Feldman-Cousins tables [9]. The expected background $n_{b}$ was determined from the extrapolation of the L0 hits distribution of MC muons, normalized to the data distribution, while $n_{s}$ is given by the number of nuclearite events surviving the cuts.
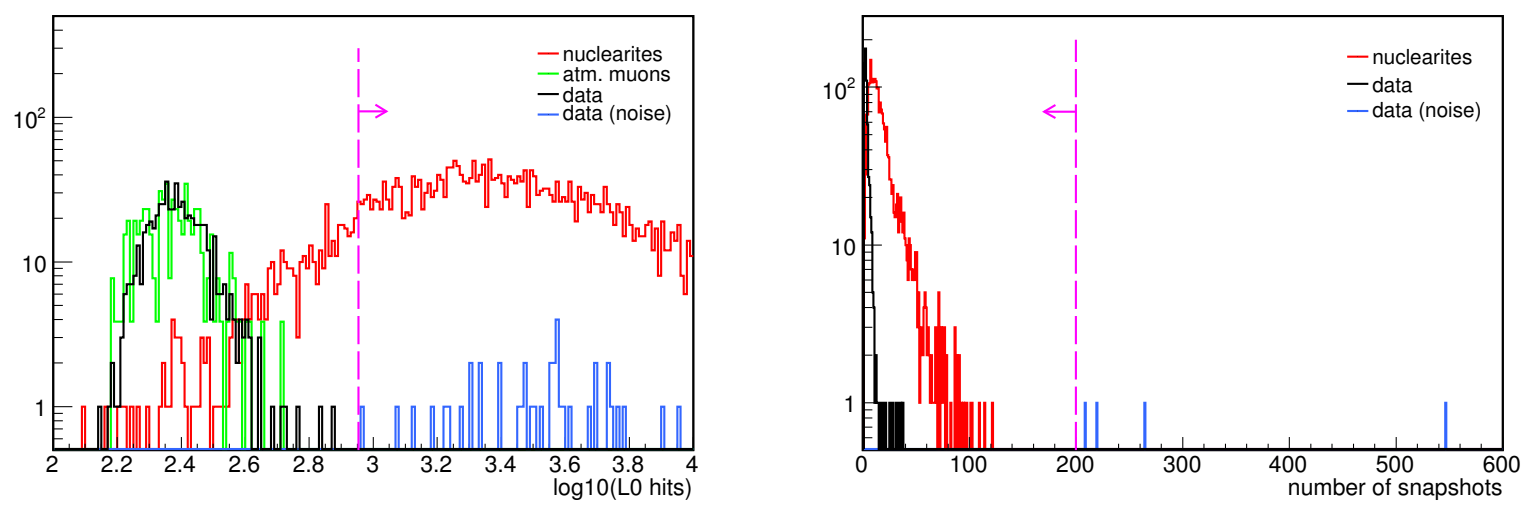

Figure 2: Left: Distribution of the number of L0 hits for MC nuclearites, muons and data snapshots. Snapshots with large values in data are shown with blue line, and are rejected by the $\mathrm{C} 2 \mathrm{a}$ cut presented in the right-hand side plot. The optimized $\mathrm{C} 2 \mathrm{~b}$ cut on the number of L0 hits is shown with a vertical dashed line. Right: Distribution of snapshots per event for nuclearites, and snapshots per frame for data; noisy frames are represented with blue line. A selection cut at 200 snapshots (C2a) rejects the bioluminescence contribution.

The value of the cut on the number of $\mathrm{L} 0$ hits, denoted $\mathrm{C} 2 \mathrm{~b}$, is chosen for the minimum MRF 
obtained for nuclearites. The selection condition requires that the number of $\mathrm{L} 0$ hits in a snapshot to be larger than 900, as shown in the left-hand side of Figure 2. After applying the second level cut, no MC muon or data snapshots survived.

As a final step in the candidate event identification, the surviving snapshots were used to look for other snaphots around them in a time interval of $\sim 1 \mathrm{~ms}$, i.e. the time a particle of velocity $\beta \simeq 10^{-3}$ crosses the detector. If found, the sequences of snapshots are reconstructed as events.

\section{Results}

The results of the cuts applied to the MC nuclearite and muon samples, as well as to data sample are shown in Table 1.

\begin{tabular}{lccccc}
\hline sample & snapshots & after & after & after & reconstructed \\
& & C1 cuts & C2a cut & C2b cut & events \\
\hline nuclearites & 36403 & 5626 & 5626 & 5190 & 2254 \\
MC muons & 2431379 & 152 & 152 & 0.0065 & 0 \\
data & 9135988 & 628 & 587 & 0 & 0 \\
\hline
\end{tabular}

Table 1: The number of snapshots in each sample, as well as the remaining snapshots after the first level (C1) cuts and second level (C2a and $\mathrm{C} 2 \mathrm{~b}$ ) cuts were applied to the data and $\mathrm{MC}$ samples, are given. The last column shows the number of reconstructed events remaining in the studied samples.

In order to calculate the detector sensitivity to nuclearites, the Feldman-Cousins prescription [9] was used, considering events with a Poisson distribution:

$$
\phi_{90}=\frac{\bar{\mu}_{90 \%}}{A \times T},
$$

where $A$ is the detector acceptance, and $T$ the live time.

The effective acceptance $A$ of ANTARES to a downgoing flux of nuclearites is computed for each simulated mass as follows:

$$
A=S \times \frac{N_{\text {nucl }}}{N_{\text {sim }}},
$$

where $S$ is the area of the simulation hemisphere and $N_{n u c l} / N_{\text {sim }}$ is the ratio of the number of nuclearite events that passed the selection cuts to the number of simulated events.

The sensitivity expected from the analysis of $\sim 159$ days of data taken in 2009 is shown in Figure 3. The ANTARES preliminary sensitivity is compared with previous limits from the MACRO [2] and SLIM [3] experiments and with the ANTARES upper limits obtained from the analysis of data taken in 2007 and 2008 [4].

\section{Conclusions}

A new analysis was developed for the search of nuclearites with the ANTARES detector, using data taken in 2009. While most of the background was removed after applying cuts on the reconstructed track parameters, hints of bursts of high bioluminescence activity were observed in the remaining data events. With these events rejected based on the noise level in their related frames, a final optimized cut allowed to reject the background and calculate the detector sensitivity. 


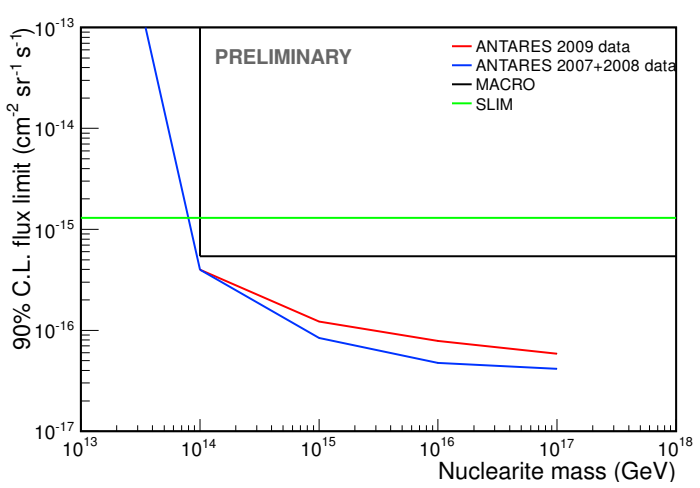

Figure 3: Sensitivity of ANTARES to a flux of downgoing nuclearites, using 159 days of data taken in 2009.

Preliminary results are comparable to the upper limits for a downgoing nuclearite flux, obtained in a previous analysis of the 2007 and 2008 data [4]. Further improvement of the sensitivity and upper limits can be achieved by extending the search to the next years of ANTARES data.

\section{References}

[1] E. Witten, Cosmic separation of phases, Phys. Rev. D 30 (1984) 272.

[2] M. Ambrosio et al., Nuclearite search with the MACRO detector at Gran Sasso, Eur. Phys. J. C 13 (2000) 453.

[3] S. Cecchini et al., Results of the search for strange quark matter and Q-balls with the SLIM experiment, Eur. Phys. J. C 57 (2008) 525.

[4] G. E. Păvălaş, (ANTARES Collaboration), Search for massive exotic particles with the ANTARES neutrino telescope, in proceedings of The 23rd European Cosmic Ray Simpozium, Moscow, Russia 409 (2013) 012135.

[5] E. Farhi, Strange matter, Phys. Rev. D 30 (1984) 2379.

[6] A. De Rujula and S. L. Glashow, Nuclearites - a novel form of cosmic radiation, Nature 312 (1984) 734

[7] G. Carminati at al., Atmospheric MUons from PArametric formulas: a fast GEnerator for neutrino telescopes, Comput. Phys. Commun. 179 (2008) 915.

[8] G. C. Hill and K. Rawlins, Unbiased cut selection for optimal upper limits in neutrino detectors: the model rejection potential technique, Astropart. Phys. 19 (2003) 393.

[9] G. J. Feldman and R.D. Cousins, A unified approach to the classical statistical analysis of small signals, Phys. Rev. D 57 (1988) 3873. 